\title{
Ultra-Compact Photonic Crystal Based Water Temperature Sensor
}

\author{
Mahmoud NIKOUFARD*, Masoud KAZEMI ALAMOUTI, and Alireza ADEL \\ Department of Electronics, Faculty of Electrical and Computer Engineering, University of Kashan, Kashan, Iran \\ *Corresponding author: Mahmoud NIKOUFARDＥ-mail: mnik@kashanu.ac.ir
}

\begin{abstract}
We design an ultra-compact water temperature sensor by using the photonic crystal technology on the InP substrate at the $1.55-\mu \mathrm{m}$ wavelength window. The photonic crystal consists of rods in a hexagonal lattice and a polymethyl methacrylate (PMMA) background. By using the plane wave expansion (PWE) method, the lattice constant and radius of rods are obtained, $520 \mathrm{~nm}$ and $80.6 \mathrm{~nm}$, respectively. With a nanocavity placed in the waveguide, a resonance peak is observed at the $1.55-\mu \mathrm{m}$ wavelength window. Any change of the water temperature inside the nanocavity results in the shift of the resonance wavelength. Our simulations show a shift of about $11 \mathrm{~nm}$ for a temperature change of $22.5^{\circ} \mathrm{C}$. The resonance wavelength has a linear relation with the water temperature.
\end{abstract}

Keywords: Sensor; water temperature; InGaAsP material; photonic crystal

Citation: Mahmoud NIKOUFARD, Masoud KAZEMI ALAMOUTI, and Alireza ADEL, "Ultra-Compact Photonic Crystal Based Water Temperature Sensor," Photonic Sensors, 2016, 6(3): 274-278.

\section{Introduction}

Photonic crystals are based on reflection and transmission of light in a periodic structure. This structure is used to avoid the propagation of electromagnetic wave in a certain frequency band, which is called photonic bandgap [1]. This properties and other capabilities like high flexibility design in the nanometer scale and integration with photodetector and laser, have turned photonic crystals to one of the important fields in the nano-scale researches [2-5]. One of the impressive factors for the photonic crystal lattice is the refractive index [6]. Meanwhile, the refractive index is one of the important parameters to identify gases and liquids. Many factors influence the refractive index such as temperature and pressure [7,8]. Considering the fact that temperature variation influences the refractive index [9] and the refractive index influences the output wavelength in a photonic crystal lattice, the temperature can easily be determined. In this paper, a water temperature sensor is designed based on photonic crystals with InGaAsP materials and a hexagonal lattice. The background is filled with polymethyl methacrylate (PMMA), and then by making a linear defect in photonic crystals, a path for the propagation of light has been created at the $1.55-\mu \mathrm{m}$ wavelength. Existence of a large cavity defect in the light path makes a space for water to cross the sensor. One of the advantages of this sensor is that it can be monolithically integrated with a laser source and a photodetector having an ultra-compact water temperature sensor. In addition, the material safety has to be considered to prevent

Received: 2 February 2016 / Revised: 3 June 2016

(C) The Author(s) 2016. This article is published with open access at Springerlink.com

DOI: $10.1007 / \mathrm{s} 13320-016-0321-0$

Article type: Regular 
water pollution.

\section{Structure of photonic crystal}

The schematic of photonic crystal with a hexagonal lattice and a pillar-based structure is depicted in Fig. 1. The layer stack consists of a film layer of InGaAsP with an energy bandgap of $E_{g}=0.992 \mathrm{eV}\left(\lambda_{g}=1.25 \mu \mathrm{m}\right)$ and a thickness of $500 \mathrm{~nm}$, denoted as $\mathrm{Q}(1.25)$, and an upper cladding layer of InP with a thickness of $1.5 \mu \mathrm{m}$ on the InP substrate. The refractive indices of the InP cladding and InGaAsP [Q(1.25)] film layer are 3.17 and 3.3640, respectively, at the $1.55-\mu \mathrm{m}$ wavelength. The refractive index of the $\mathrm{Q}(1.25)$ film layer is higher than that of the lower cladding and substrate layers $[10,11]$. Specifications of layers including the diameter $(r)$, lattice constant $(a)$, and refractive index $(n)$ are depicted in Fig. 1.
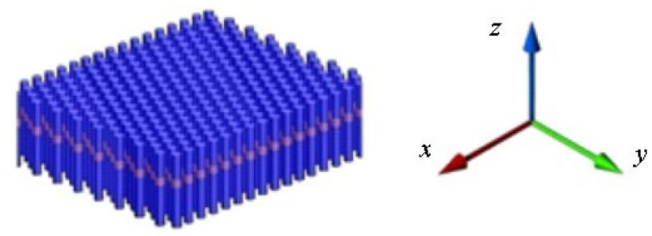

(a)

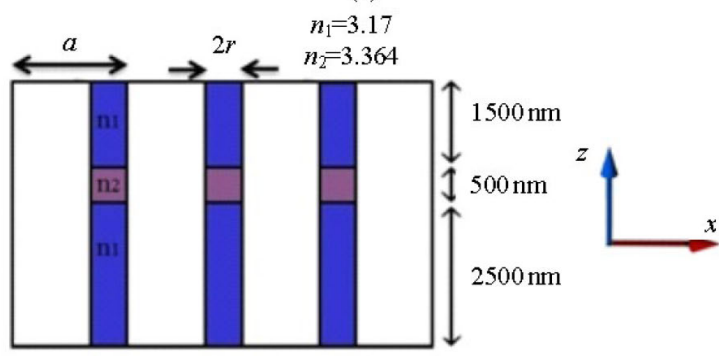

(b)

Fig. 1 Specifications of layers: (a) 3D-geometry of photonic crystal and (b) $x-z$ view with layer specifications.

Due to the memory and speed limitations of computational computer, the three-dimensional (3D) simulation of the sensor is reduced to a two-dimensional (2D) photonic crystal structure. At first, the effective refractive index of layers is determined to be 3.2634 using COMSOL software. The two-dimensional plane wave expansion method is used to determine the photonic bandgap of the dielectric rods periodically arranged in the hexagonal lattice for the polarization of the E-field parallel and perpendicular to the dielectric rods (TM and TE modes, respectively). The photonic bandgap diagram for the different radii is plotted in Fig. 2 for two polarization states of TE and TM modes when the radius varies within a $0 \mathrm{~nm}-250 \mathrm{~nm}$ range with a lattice constant of $520 \mathrm{~nm}$. It can be seen that for a rod with a radius of $86 \mathrm{~nm}$, the band gap includes the normalized frequency of 0.3354 equivalent to a wavelength of $1.55 \mu \mathrm{m}$. In Fig. 3, the band structure is shown in the $\mathrm{K}$ direction for both TE and TM modes.

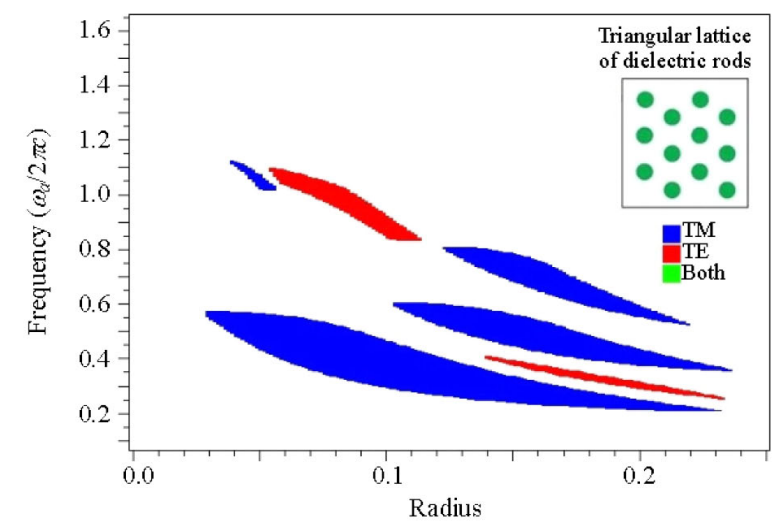

Fig. 2 Normalized frequency bandgap as a function of radius $(\mu \mathrm{m})$ for TE and TM polarization modes.

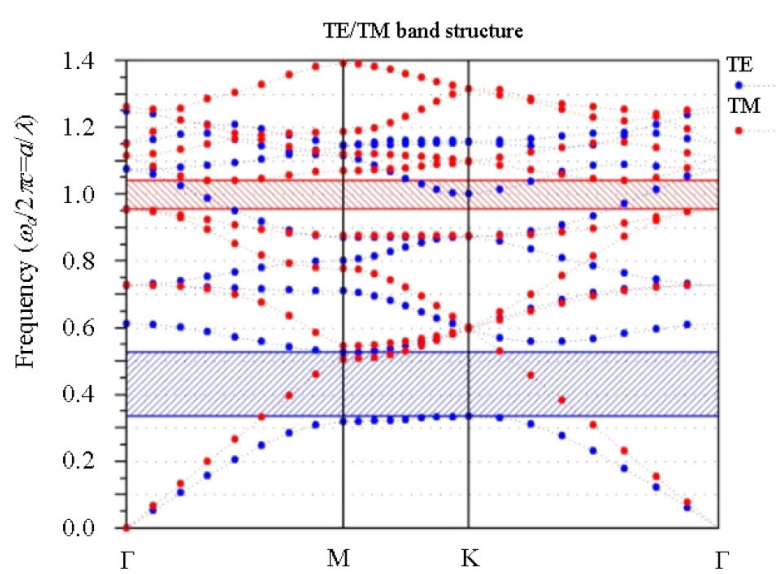

Fig. 3 Band structure in the $\mathrm{K}$ direction as a function of the normalized frequency for TE and TM modes.

The main advantage of this layer stack is that it can be integrated with active devices such as the laser and photodetector, as can be seen in Fig. 4. The on-chip integration of the nanosensors, laser source, and photodetector is attractive because of the higher 
operating temperature of InP-based devices, the possibility of making very compact sensor through the on-chip integration of optical transmitter, and receiver components with the photonic crystal-based sensor.

The laser produces a light wave at the $1.55-\mu \mathrm{m}$ wavelength launching into the photonic crystal sensor. The light is then detected by the photodetector. The only difference between the water temperature sensor (passive structure) and the laser source and photodetector (active structures) is a layer of InGaAsP with a bandgap wavelength of $1.55 \mu \mathrm{m}$ [denoted as $\mathrm{Q}(1.55)]$. This layer has a direct bandgap at the $1.55-\mu \mathrm{m}$ wavelength and a bandgap energy of lower than that of adjacent layers. A direct bias voltage applied to the laser source injects an electron-hole pair to the active layer of $\mathrm{Q}(1.55)$, generating the light beam. Whereas, a reversed bias voltage applied to the photodetector converts the absorbed light to the electron-hole pair in the active layer (shown in Fig. 4). This scheme requires a butt-joint technology to fabricate the active and passive structures on a single chip [10, 11].

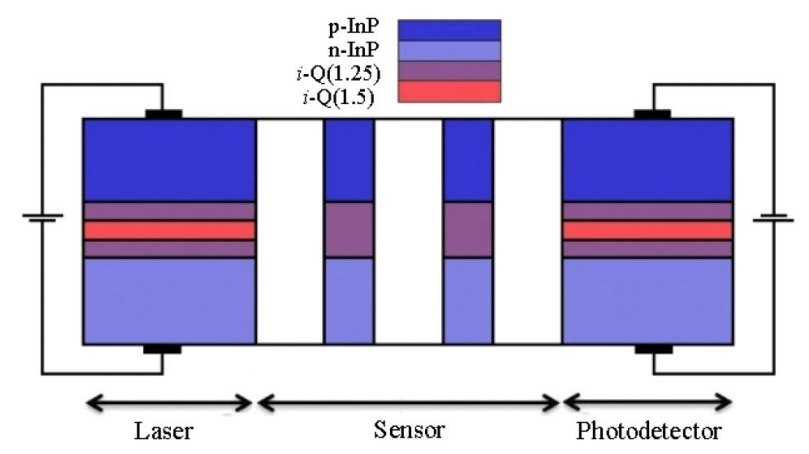

Fig. 4 Schematic view of the water temperature sensor chip including the monolithic integrated laser, photonic-crystal-based sensor, and photodetector.

\section{Photonic crystal based water temperature sensor}

The photonic crystal-based temperature sensor has an area of $17 \times 17$ lattice constants in a hexagonal lattice dielectric-pillar. The background is filled with the PMMA material having a refractive index of 1.4914. By creating a line defect and a great hole with a radius of $1.05 \mu \mathrm{m}$, we have developed a sensor that works at the wavelength window of $1.55 \mu \mathrm{m}$. The great hole acts as a resonator allowing water to enter the sensor, which shifts the resonance wavelength of resonator. In addition, the resonance wavelength depends on the water temperature.

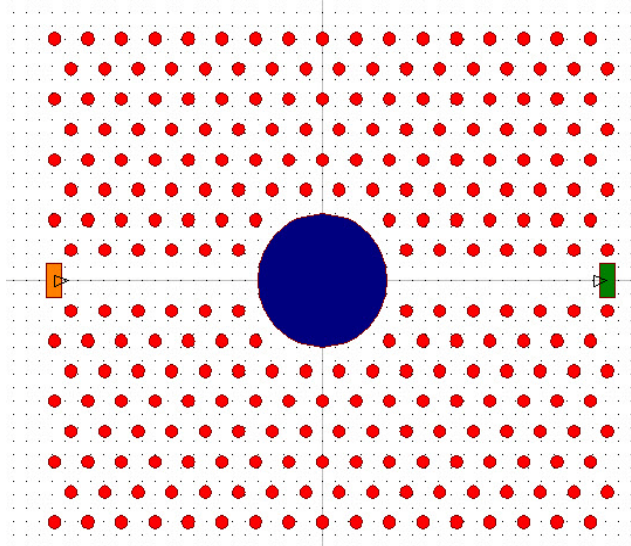

Fig. 5 Photonic crystal structure after creating the line defect (the large cavity in the center of the sensor acts as a resonator).

\section{Simulation results}

The water temperature sensor is investigated using the finite difference time domain (FDTD) method. Figure 6 shows the field distribution in the photonic crystal sensor at a wavelength of $1.55 \mu \mathrm{m}$. A pulsed laser beam is launched toward the temperature sensor. The optical signal is then propagated through the defects and absorbed in the photodetector after the photonic crystal-based sensor. The change in the water temperature causes a change in the refractive index of the large hole, thus resulting in a shift in the resonant wavelength peak. The relationship between the water temperature and refractive index at a pressure of 1 bar is shown in Fig. 7 [6]. Multiple simulations are performed at several temperatures to verify the resonant wavelength peak. The simulation results are presented in Table 1 (see also Fig. 8). It can be observed that the relationship between the water temperature and the resonant wavelength peak is linear in the wide range of $24{ }^{\circ} \mathrm{C}$ to $55^{\circ} \mathrm{C}$. 
Table 1 Relationship among the resonant wavelength peak, refractive index, and temperature of water in the water temperature sensor.

\begin{tabular}{ccc}
\hline Temperature $\left({ }^{\circ} \mathrm{C}\right)$ & Refractive index & Wavelength $(\mathrm{nm})$ \\
\hline 24.8 & 1.33293 & 1578.888 \\
34.5 & 1.33177 & 1578.303 \\
40 & 1.3302 & 1572.427 \\
47 & 1.3290 & 1569.268 \\
54.34 & 1.32866 & 1567.132 \\
\hline
\end{tabular}

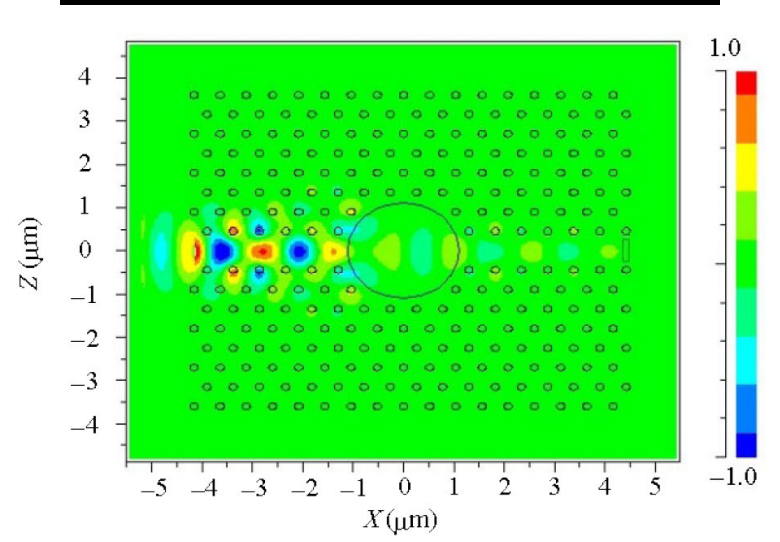

Fig. 6 Electric field distribution in the photonic crystal-based water temperature sensor at the $1.55-\mu \mathrm{m}$ wavelength.

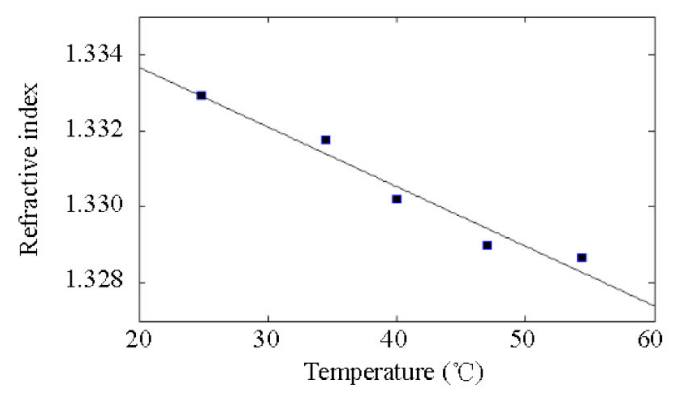

Fig. 7 Refractive index of water as a function of the water temperature with a linear curve fit of $n=-0.00015 T+1.3367$.

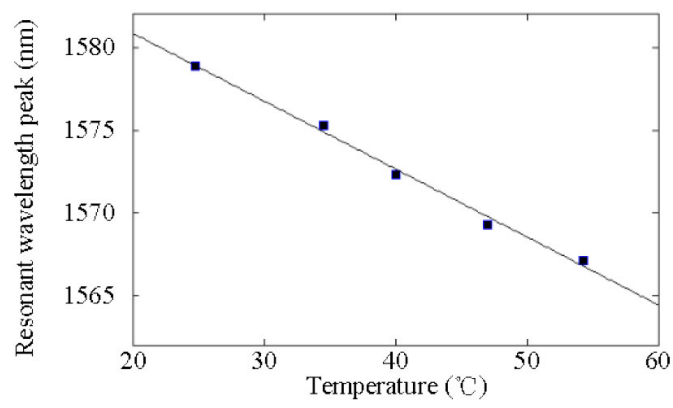

Fig. 8 Resonant wavelength peak as a function of temperature with a linear curve fit of $\lambda=-0.41083 T+1589.1$.

\section{Conclusions}

In this article, we design an ultra-compact water temperature sensor by using the photonic crystal technology. The main advantage of the presented structure is the capability of monolithic integration with the laser and photodetector by using the butt-joint technology which reduces the size of the sensor to nano-scale. Simulations show that there is a linear relationship between the water temperature and the resonant peak wavelength of the sensor. A water temperature change in the range of $24.8{ }^{\circ} \mathrm{C}$ to $54.34{ }^{\circ} \mathrm{C}$ causes a wavelength shift of about $10 \mathrm{~nm}$.

Open Access This article is distributed under the terms of the Creative Commons Attribution 4.0 International License (http://creativecommons.org/ licenses/by/4.0/), which permits unrestricted use, distribution, and reproduction in any medium, provided you give appropriate credit to the original author(s) and the source, provide a link to the Creative Commons license, and indicate if changes were made.

\section{References}

[1] J. D. Joannopoulos, S. G. Johnson, J. N. Winn, and R. D. Meade, "Photonic crystals: molding the flow of fight," Computing in Science and Engineering, 1995, 3(6): 38-47.

[2] S. Olyaee and F. Taghipour, "Design of new square-lattice photonic crystal fibers for optical communication applications," International Journal of Physical Sciences, 2011, 6(18): 4405-4411.

[3] S. Olyaee and F. Taghipour, "Ultra-flattened dispersion hexagonal photonic crystal fibre with low confinement loss and large effective area," IET Optoelectronics, 2012, 6(2): 82-87.

[4] C. Kang and S. M. Weiss, "Photonic crystal with multiple-hole defect for sensor applications," Optics Express, 2008, 16(22): 18188-18193.

[5] S. Hosseini, M. Haas, D. Plettemeier, and K. Jamshidi, Integrated optical devices for $3 D$ photonic transceivers. Germany: Springer International Publishing, 2016.

[6] T. Stomeo, M. Grande, A. Qualtieri, A. Passaseo, A. Salhi, M. De Vittorio, et al., "Fabrication of force sensors based on two-dimensional photonic crystal technology," Microelectronic Engineering, 2007, 84(5-8): 1450-1453.

[7] R. M. Waxler and C. E. Weir, "Effect of pressure and temperature on the refractive indices of benzene, carbon tetrachloride and water," Biochemical \& Biophysical Research Communications, 1973, 51(2): 
$163-171$.

[8] S. Tao, D. Chen, J. Wang, J. Qiao, and Y. Duan, "A high sensitivity pressure sensor based on two-dimensional photonic crystal," Photonic Sensors, 2016, 6(2): 137-142.

[9] H. Wang, H. Meng, R. Xiong, Q. Wang, B. Huang, X. Zhang, et al., "Simultaneous measurement of refractive index and temperature based on asymmetric structures modal interference," Optics Communications, 2016, 364: 191-194.
[10] L. Xu, M. Nikoufard, X. J. Leijtens, T. De Vries, E. Smalbrugge, R. Nötzel, et al., "High-performance InP-based photodetector in an amplifier layer stack on semi-insulating substrate," IEEE Photonics Technology Letters, 20(23): 1941-1943.

[11] K. A. Williams, E. A. J. M. Bente, D. Heiss, Y. Jiao, K. Lawniczuk, X. J. M. Leijtens, et al., "InP photonic circuits using generic integration [Invited]," Photonics Research, 2015, 3(5): B60-B68. 